\title{
Growth polarity transitions in a dimorphic fission yeast
}

\author{
M. Sipiczki, ${ }^{1,2}$ K. Takeo ${ }^{3}$ and A. Grallert ${ }^{1}$ \\ Author for correspondence: M. Sipiczki. Tel: +3652316 666. Fax: + 3652348550. \\ e-mail: lipovy@tigris.klte.hu
}

\footnotetext{
1,2 Department of Genetics 1 and Institute of Biology2, University of Debrecen, PO Box 56, H-4010 Debrecen, Hungary

3 Division of Ultrastructure and Function, Research Center for Pathogenic Fungi and Microbial Toxicoses, Chiba University, Chiba, Japan
}

\begin{abstract}
Fission yeast cells grow by extension at the ends (poles) and divide by transverse fission. It has previously been reported that Schizosaccharomyces japonicus var. japonicus can switch to unipolar, filamentous growth. Here it is shown that the yeast-to-mycelium transition is a gradual process involving a changeover to unipolar growth associated with asymmetric divisions, the development of large polarly located vacuoles, the modifications of the actin and microtubular cytoskeleton and the repression of cell separation after division. High concentrations of glucose in the medium or supplementation of the medium with caffeine or CAMP support the bipolar yeast phase, inhibit the transition to the mycelial phase and induce the conversion of hyphae to yeasts. These effects suggest that CAMP may be involved in the regulation of dimorphism. Temperatures below $18^{\circ} \mathrm{C}$ or over $35^{\circ} \mathrm{C}$ are restrictive for the mycelial phase and provoke a return to yeast phase.
\end{abstract}

Keywords: Schizosaccharomyces, dimorphism, polarity, vacuolation, cAMP

\section{INTRODUCTION}

Cells respond to their environments in a variety of ways: by starting or stopping growth and the division cycle, by embarking on a new developmental course or by varying the level of expression of particular genes. One of the outstanding problems of cell growth, division and differentiation is the establishment and maintenance of cell polarity. The unicellular budding yeast Saccharomyces cerevisiae and the fission yeast Schizosaccharomyces pombe are excellent models to study cell polarity for several reasons. First, the cells of these yeasts have very regular although rather different shapes and grow in a highly polarized fashion. Second, cell cycle regulation of the cytoskeleton is well-documented. Third, both yeasts are convenient for genetic analysis and amenable to manipulation with recombinant DNA techniques.

An advantage of Sacch. cerevisiae is its ability to change its growth morphology in response to environmental stimuli. It has long been known that its cells can form pseudomycelial chains on certain media (e.g. Yarrow, 1984). The phenomenon has recently been rediscovered by molecular biologists and exploited for the investigation of the determination and regulation of cell polarity. It has turned out that the diploid cells of Sacch.

Abbreviations: BEG, 'both-end-growing'; DAPI, 4',6-diamidino-2phenylindole; NEG, 'new-end-growing'; OEG, 'old-end-growing'. cerevisiae can undergo a switch to a pseudohyphal form in response to nitrogen starvation (Gimeno et al., 1992). The haploid cells respond to nutrient deprivation by penetrating the agar, a process termed the 'invasive growth response', which is characterized by the capacity to grow into the agar (Roberts \& Fink, 1995). This invasive growth behaviour requires a switch from an axial to a bipolar mode of bud site selection. The morphological transitions of Sacch. cerevisiae make use of the function of a MAP kinase module which is also involved in the pheromone signalling pathway (for a review see Madhani \& Fink, 1998).

There is little information about hyphal development in Schiz. pombe: Multiseptate hyphal cells were occasionally observed in nutrient-limited chemostat cultures (McDonald \& Tsai, 1989) and in cultures supplemented with aniline blue (Pancaldi et al., 1989). We reported that the mutation sep1-1 renders the cells unable to separate after septation, which changes the morphology to filamentous. In contrast to the filaments (pseudohyphae) of Sacch. cerevisiae, these structures are true hyphae. Both the tip cells and the internal cells of the hyphae can grow, which results in the formation of branches. Each internal cell develops two branches, suggesting that the mutation does not affect the bipolar mode of growth (Sipiczki et al., 1993). Occasional formation of branched filaments can also be provoked by non-lethal mutations in a number of other genes, including those of the sep family (Grallert et al., 1997) 
and the stress-activated MAP kinase gene spm1 (Zaitsevskaya-Carter \& Cooper, 1997). The occurrence of bipolarly growing cells in the hyphae suggests that neither of these mutants are defective in polarized growth.

The related Schizosaccharomyces japonicus var. japoni$c u s$ is a dimorphic fission yeast capable of producing alternate morphological forms in response to environmental changes (Sipiczki et al., 1998). It exhibits yeastto-mycelium dimorphism, i.e. the ability to produce a filamentous form which either grows on the surface of the substrate as pseudomycelium or penetrates the substrate and forms true mycelium. The hyphae grow unipolarly and due to the enlargement of vacuoles at the non-growing pole, their extension is much more effective than that of the yeast cells. The mycelium can convert into arthroconidia or return to the yeast phase. The environmentally controlled transitions between the yeast phase and the mycelial phase provide a novel system to study cell polarity and polar growth in fission yeasts. In a recent paper (Sipiczki et al., 1998) we described the dimorphic cycle and characterized the morphotypes. Here we report that the yeast-to-mycelium transition is a gradual process associated with a switch from bipolar growth to unipolar growth, a transition from symmetric to asymmetric division, the repression of cell separation (septum cleavage), the development of large vacuoles at the non-growing pole and the reorganization of the actin and tubulin cytoskeleton. High concentrations of glucose, caffeine and cAMP in the medium inhibit the yeast-to-mycelium transition and can induce the mycelium-to-yeast transition, suggesting that cAMP may be involved in the regulation of dimorphism. The phase transitions of Schiz. japonicus var. japonicus open new perspectives for the investigation of polarity in fission yeasts.

\section{METHODS}

Strain and media. Schizosaccharomyces japonicus var. japonicus Yukawa et Maki CCY-44-5-1 (CBS 354), a dimorphic fission yeast, was obtained from the Czechoslovak Collection of Yeasts (CCY), Bratislava, Slovakia.

The media YEA, YEL and YEMA were described by Sipiczki \& Ferenczy (1977) and Sipiczki et al. (1998). YEG was as YEA but solidified with $15 \%(\mathrm{w} / \mathrm{v})$ gelatin instead of agar. All cultures were incubated at $30^{\circ} \mathrm{C}$, unless indicated otherwise.

Time-lapse microphotography and fluorescence microscopy. Time-lapse experiments and photography were performed as described by Sipiczki et al. (1993). Calcofluor was used to stain cell walls and septa (Johnson et al., 1979). The staining procedure using 4',6-diamidino-2-phenylindole (DAPI) to visualize nuclei and the procedure using rhodamine-conjugated phalloidin to visualize actin were as described previously (Alfa et al., 1993). Tubulin was stained as described by Hagan \& Hyams (1988). The primary antibody was the TAT1 anti- $\alpha$-tubulin mouse monoclonal antibody (a gift from Keith Gull, University of Manchester, UK). An FITC-conjugated goat anti-mouse IgG antibody (Sigma F-0257) was used as secondary antibody. To stain hyphae, small blocks were cut from the invasive mycelium grown in YEG and collected in a glass centrifuge tube. The tube was filled up to
$10 \mathrm{ml}$ with YEL and incubated in a water bath at $35^{\circ} \mathrm{C}$ until the gelatin had melted (10-15 min). The mycelium was then collected by centrifugation and after removing the supernatant the pellet was fixed. Vacuoles were stained with the yeast vacuole marker sampler kit Y-7531, component B, Cell Tracker Blue CMAC 7-amino-4-chloromethylcoumarin (Molecular Probes) according to the manufacturer's instruction manual. The concentration applied was $100 \mu \mathrm{M}$. Cells were viewed and photographed using an Olympus $\mathrm{BH}-2$ microscope.

\section{RESULTS}

\section{Transition from unipolar to bipolar growth on solid medium}

Yeast cells of CCY-44-5-1 cultivated in the liquid medium YEL or on the solid media YEA and YEMA showed a uniform fission yeast morphology in the earlyexponential phase of growth (Fig. 1a). In liquid cultures this morphology remained characteristic even in stationary phase. On the solid media, however, the cells underwent drastic changes as the cultures reached the late-exponential phase (Fig. 1b). They became heterogeneous both in size and in shape. The older the culture was the more cells were highly elongated and irregularly bent. Short chains composed of 2-10 cells were also visible.

To investigate cell elongation, we stained the cells with calcofluor, a dye widely used in Schiz. pombe to visualize cell wall regions of different origins (Johnson et al.,

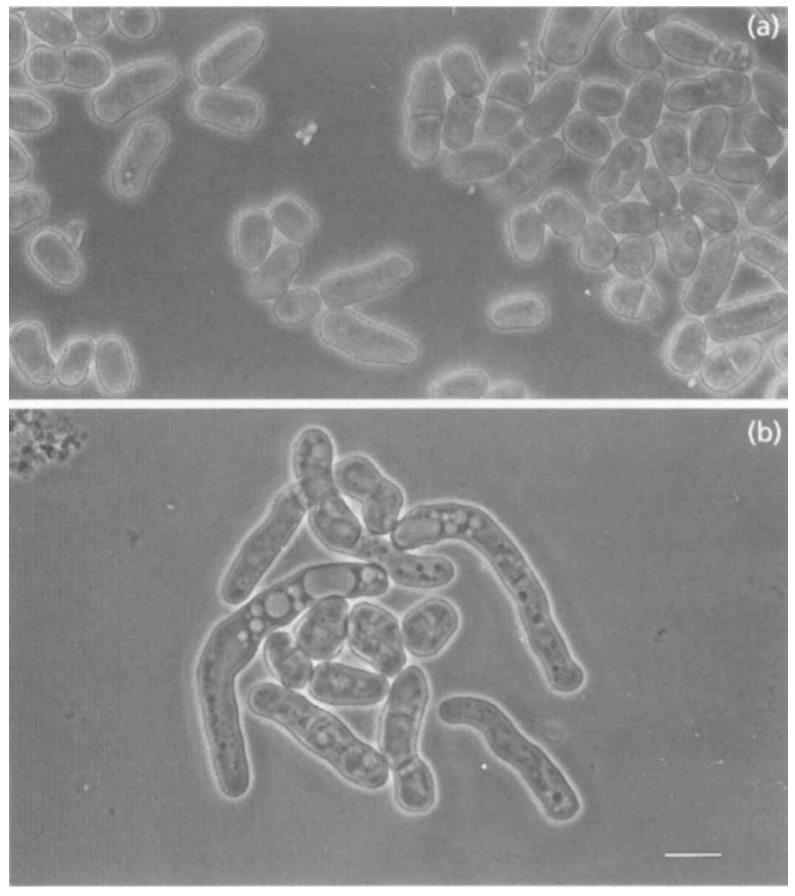

Fig. 1. Morphology of Schiz. japonicus var. japonicus. (a) Cells of an overnight (exponential-phase) culture grown in liquid medium (YEL). (b) Cells of a 3-d-old (early-stationary-phase) culture grown on agar medium (YEA). Bar, $5 \mu \mathrm{m}$. 

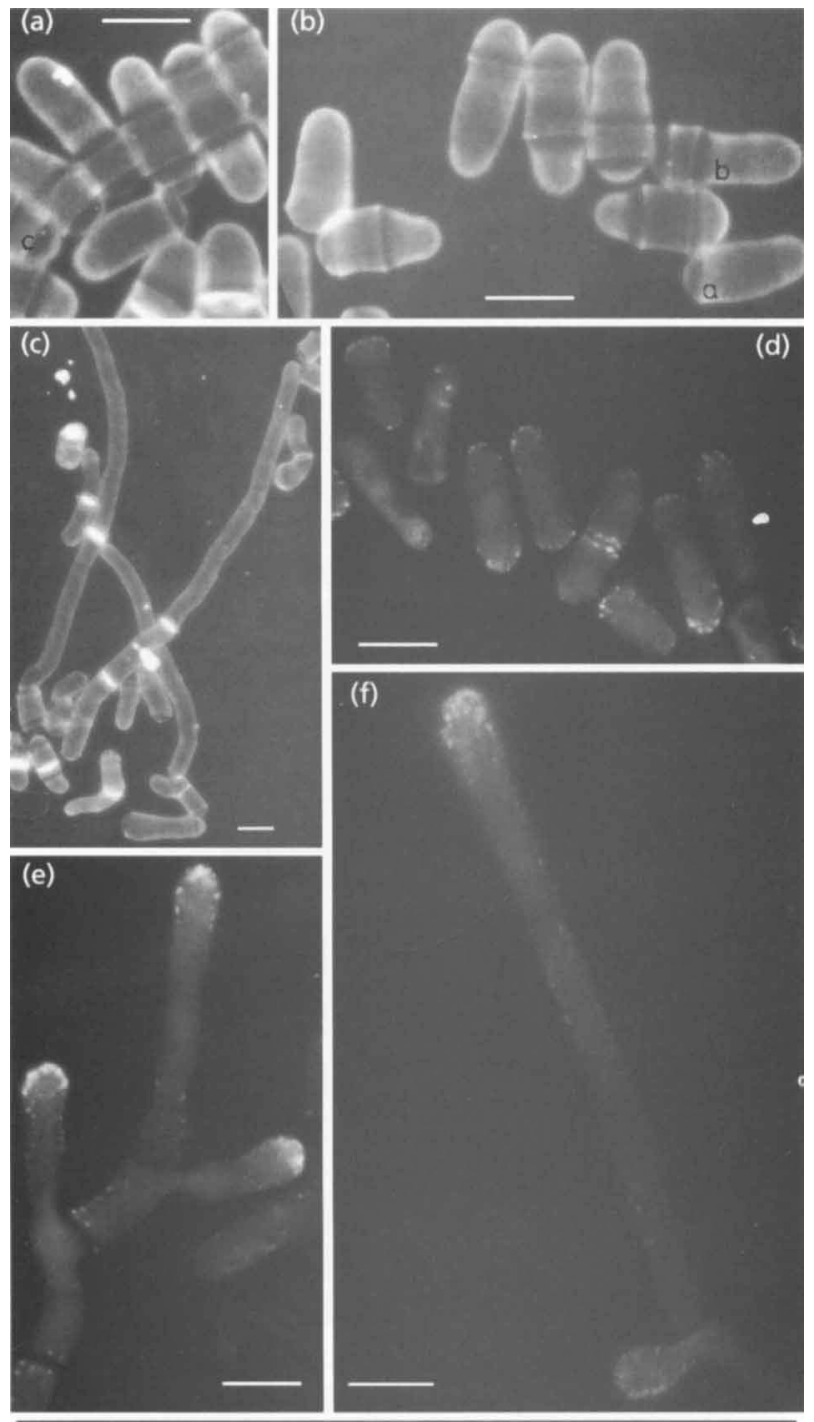

(g)

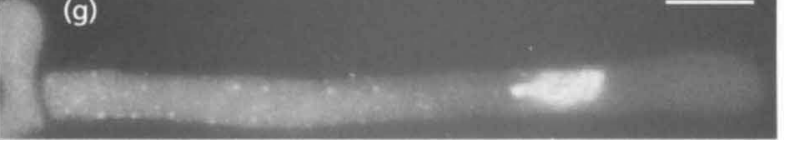

Fig. 2. Polar growth patterns. Calcofluor-stained cells of (a) an overnight (exponential-phase) culture grown in liquid medium, (b) an overnight (exponential-phase) culture grown on agar medium and (c) a 3-d-old (early-stationary-phase) culture grown on agar medium. Also shown is actin distribution (d) in yeasts of an overnight culture grown in liquid medium, (e) in an elongated yeast cell from a 2-d-old agar culture and ( $f$ ) in an invasive hypha. $(\mathrm{g})$ Position of the nucleus in the cell shown in (f). a, 'new-born' cell; b, a 'young' cell with a single division scar; $c$, an 'experienced' cell with several division scars. Bars, $8 \mu \mathrm{m}(\mathrm{a}, \mathrm{b}, \mathrm{g}), 10 \mu \mathrm{m}(\mathrm{d}, \mathrm{e}, \mathrm{f})$ or $5 \mu \mathrm{m}(\mathrm{c})$.

1979). In a previous study we showed that calcofluor can also be used to stain the cell wall in Schiz. japonicus var. japonicus: it stained the regions developed from septal layers less intensely than the rest of the cell wall (Sipiczki et al., 1998). Here we found that the cells of the exponential-phase yeast cultures showed staining patterns that indicate bipolar growth (Fig. 2). Certain cells had one end darker than the rest of the cell. These might have been the 'new-born' cells, and the darker ends must have been wall regions formed from septa during cytokinesis. The wall regions developed from secondary septa are dark in calcofluor-stained cells of Schiz. pombe (Johnson et al., 1979). If the cell had already grown after birth at its 'new end', both of its ends were bright, but separated by a dark region, a ring-like band that had arisen from the septal region. Many cells showed alternate brighter and darker bands. These could be the 'older' or 'more experienced' cells that carried wall regions (also called division scars) inherited from several previous generations. Most of the cells had both ends bright, suggesting that they grew bipolarly. These categories were characteristic of liquid cultures in all growth phases and of the YMGA cultures during the first $20-24 \mathrm{~h}$ incubation at $30^{\circ} \mathrm{C}$.

Upon longer incubation on the solid medium, when elongated cells began to appear, a gradually increasing proportion of the population showed growth at one end only. These cells had their scars located highly asymmetrically, close to the non-growing pole (e.g. long cells in Figs $2 \mathrm{c}$ and $3 \mathrm{f}$ ). The elongated cells usually showed this pattern, indicating that their elongation was due to unipolar extension. The exact proportions of the elongated cells could not be determined because they usually formed flocks and were much more abundant in the central part than at the edges of the cultures.

The distribution of actin also indicated bipolar growth in yeast cells propagating in liquid cultures (Fig. $2 \mathrm{~d}$ ). Concentration of actin was usually detected at both poles of the cells, although scattered dots were also visible in the rest of the cell. In the dividing (septate) cells most of the actin accumulated at the septum. As the location of actin coincides with the sites of cell growth in Schiz. pombe (Marks \& Hyams, 1985), we believe that the Schiz. japonicus var. japonicus cells, which showed bipolar actin distribution, also grew bipolarly. In the elongated cells formed in agar cultures upon longer incubation, the actin dots grouped at one pole which was the growing end (Fig. 2e). In the hyphae, actin concentration was observable in the growing tips (Fig. 2f).

\section{Asymmetric vacuolation in unipolarly growing cells as growth silencing}

We noticed that the transition to unipolar elongation was usually associated with an intense vacuolation. The short, bipolarly growing yeast cells contained small vacuoles scattered randomly in the cytoplasm (Fig. 3a), whereas the long, unipolarly extending cells always had one or two large vacuoles besides the small ones (Fig. 3b, c). Staining these cells with calcofluor showed that the large vacuoles were located at the non-growing pole (Fig. 3e, f).

To examine the role of the vacuoles, we spread samples of 3-d-old cultures onto thin films of YEMA. This shift to the fresh medium reverts cells to the exponential 

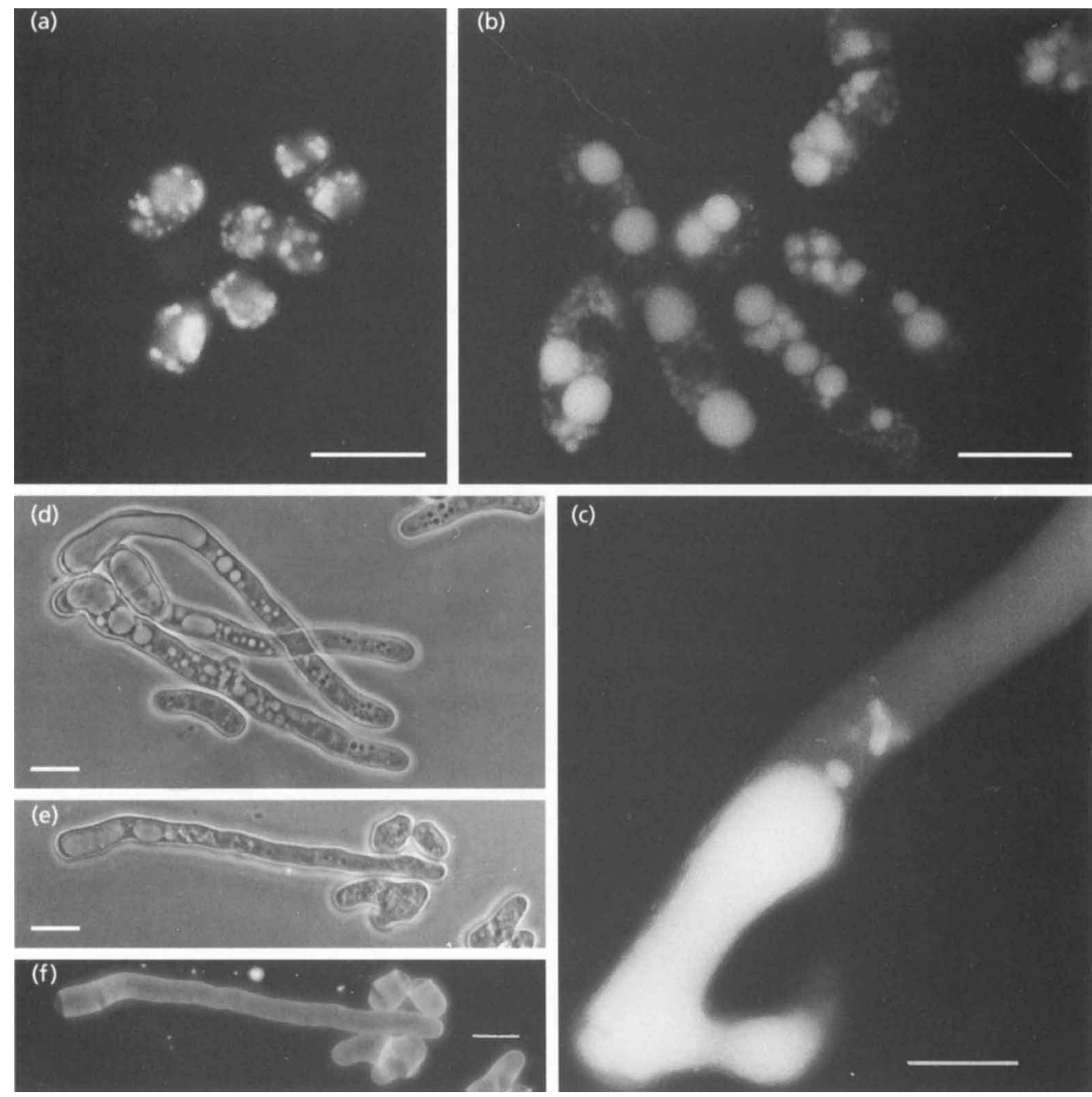

Fig. 3. Vacuolation of cells. Fluorescence image of vacuolated cells of 3-d-old cultures grown in liquid medium (a) or on agar medium ( $b, c)$. ( $d, e)$ Phase-contrast images of unipolarly growing cells from a 3-d-old culture grown on agar medium. (f) Calcofluor-stained image of the cells shown in (e). Bars, $5 \mu \mathrm{m}$.

phase of growth. We followed the process of reversion both in short and in elongated cells by time-lapse photography. The former grew at both cell ends and formed septa halfway between the ends (see short cells in Fig. 4). The latter extended unipolarly, at the nonvacuolated end, and formed septa halfway between the growing pole and the large vacuole. The septum divided the cell into a shorter yeast cell (apical daughter) and a much longer, vacuolated cell (an example is shown in Fig. 4). Two out of the 10 vacuolated cells subjected to time-lapse observation grew bipolarly. However, they did not grow at the vacuolated pole but established a new growth site at the opposite end of the vacuole and then formed lateral outgrowths (Fig. 4). Thus, the large polar vacuole appears to keep the non-growing pole silent and thus contributes to the 'fixation' of the unipolar growth pattern. In the elongated, unipolarly growing cells, the nucleus was always located closer to the growing pole (Fig. $2 \mathrm{~g}$ ), suggesting that the vacuole (which could not be preserved for photographing during fixation of the cells for staining with DAPI) isolated it from the opposite pole.

\section{Microtubular cytoskeleton}

Consistent with the suggested silencing role of the large vacuoles, no microtubules could be detected by immunofluorescence in the vacuolated parts of the unipolarly growing cells and hyphae (Fig. 5). The interphase microtubules spanned between the growing pole and the large polar vacuole at which they ended. The hyphal cells, which were much longer and also thicker than the yeasts, usually contained more microtubules than the yeast cells and formed arrays with longitudinal axes spanning between the growing tip and the large vacuole. The microtubules appeared in the stained cells as longitudinal bundles that bent where the cell was bent and also branched where the cell shape was irregular. 

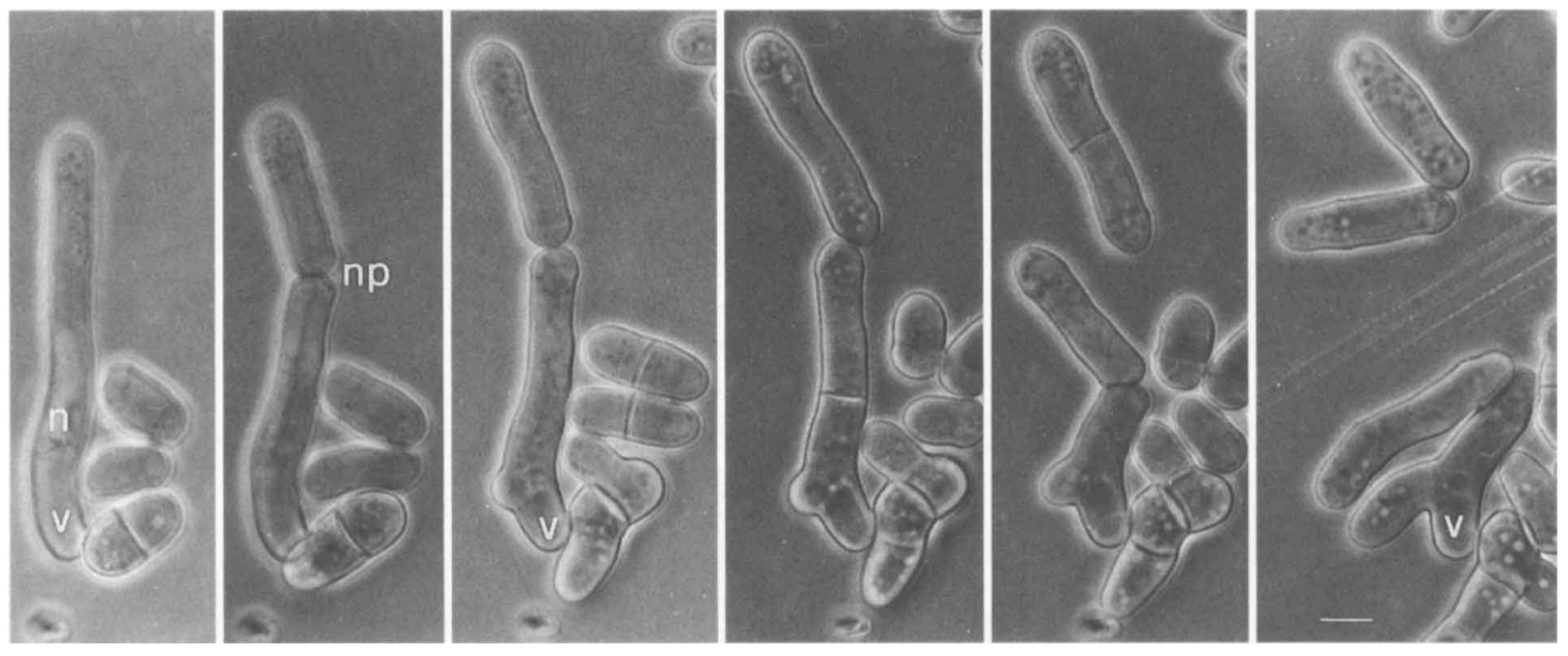

Fig. 4. Time-lapse microphotography of a vacuolated cell upon the shift onto fresh medium. Selected pictures are shown only. The photographs were taken at $0,50,95,140,155$ and $230 \mathrm{~min}$. v, Vacuole; $n$, nucleus; np, new pole for growth. Bar, $3 \mu \mathrm{m}$.

These bundles were more abundant around the nucleus, where they showed a basket-like arrangement.

\section{Vacuolation is not necessary for unipolar growth}

Next we asked if vacuolation was necessary for the transition to unipolar growth. To test this possibility, we inoculated yeast cells on YEMA plates supplemented with various concentrations of $\mathrm{KCl}$ or sorbitol. After $3 \mathrm{~d}$ incubation at $30^{\circ} \mathrm{C}$, long, vacuolated cells and cell chains were seen up to the concentrations of $0.6 \mathrm{M} \mathrm{KCl}$ and $1.1 \mathrm{M}$ sorbitol, although their morphology changed with the concentration. With the increase of the osmolality in the medium the mean cell length decreased, and more and more cells contained several small vacuoles instead of the one or two large ones seen in the control cultures (Fig. 6). However, the cell chains remained abundant even at high concentrations, suggesting that it is not the vacuolation that makes the cells grow unipolarly and form chains. Supplementation of the medium with $\mathrm{KCl}$ or sorbitol drastically inhibited the formation of the large polar vacuoles but did not abolish the transition to unipolar filamentous growth. The decrease of the cell length might then be a consequence of the failure of the cells to enlarge their vacuoles.

\section{The yeast-to-mycelium transition is sensitive to high glucose concentration and the presence of caffeine in the medium}

The osmotic pressure in the medium can also be increased by the addition of glucose. We found that this also reduced yeast elongation and vacuolation: $2.5 \%$ $(\mathrm{w} / \mathrm{v})$ glucose $(0 \cdot 126 \mathrm{M})$ drastically reduced the extent of elongation and the enlargement of vacuoles, and $10 \%$ $(\mathrm{w} / \mathrm{v})$ glucose $(0.504 \mathrm{M})$ completely blocked both processes. However, it is probably not the osmotic pressure by which glucose affects growth morphology, because these concentrations are much lower than the concentrations at which sorbitol showed a similar effect (see above).

Since glucose is known to affect the intracellular cAMP level in many fungi and yeasts, including the related Schiz. pombe (Byrne \& Hoffman, 1993), we asked if caffeine, which also interferes with the regulation of the cAMP level (e.g. Beach et al., 1985), could have a similar effect. We found that $10 \mathrm{mM}$ caffeine in YEMA inhibited the growth of yeast cells. The concentrations lower than $5 \mathrm{mM}$ had no effect on growth but inhibited the transition into the mycelial phase. Mycelium was produced only at concentrations lower than $0.5 \mathrm{mM}$ (Fig. 7). Thus, the yeast-to-hypha transition is about ten times more sensitive to caffeine than the yeast growth. Caffeine also affected the morphology of the yeast cells. The presence of $0.5 \mathrm{mM}$ of the drug in the medium dramatically reduced both the mean cell length and the degree of vacuolation; $2 \mathrm{mM}$ caffeine inhibited the enlargement of vacuoles but allowed cell chain formation and unipolar growth. Theophylline, an analogous methylxanthine that also inhibits cAMP phosphodiesterase and affects dimorphic transition in Candida albicans (Sabie \& Gadd, 1992), had no discernible effect on either phase of Schiz. japonicus up to the concentration of $5 \mathrm{mM}$. It is worth mentioning here that theophylline had no effect on the growth of Schiz. pombe up to concentrations of $6 \mathrm{mM}$ (Benko \& Sipiczki, 1992).

\section{Caffeine and CAMP induce reversion of mycelium to yeast phase}

When agar blocks containing hyphae were placed onto fresh plates, the hyphae grew into the medium only if its caffeine concentration did not exceed $0.5 \mathrm{mM}$, sug- 

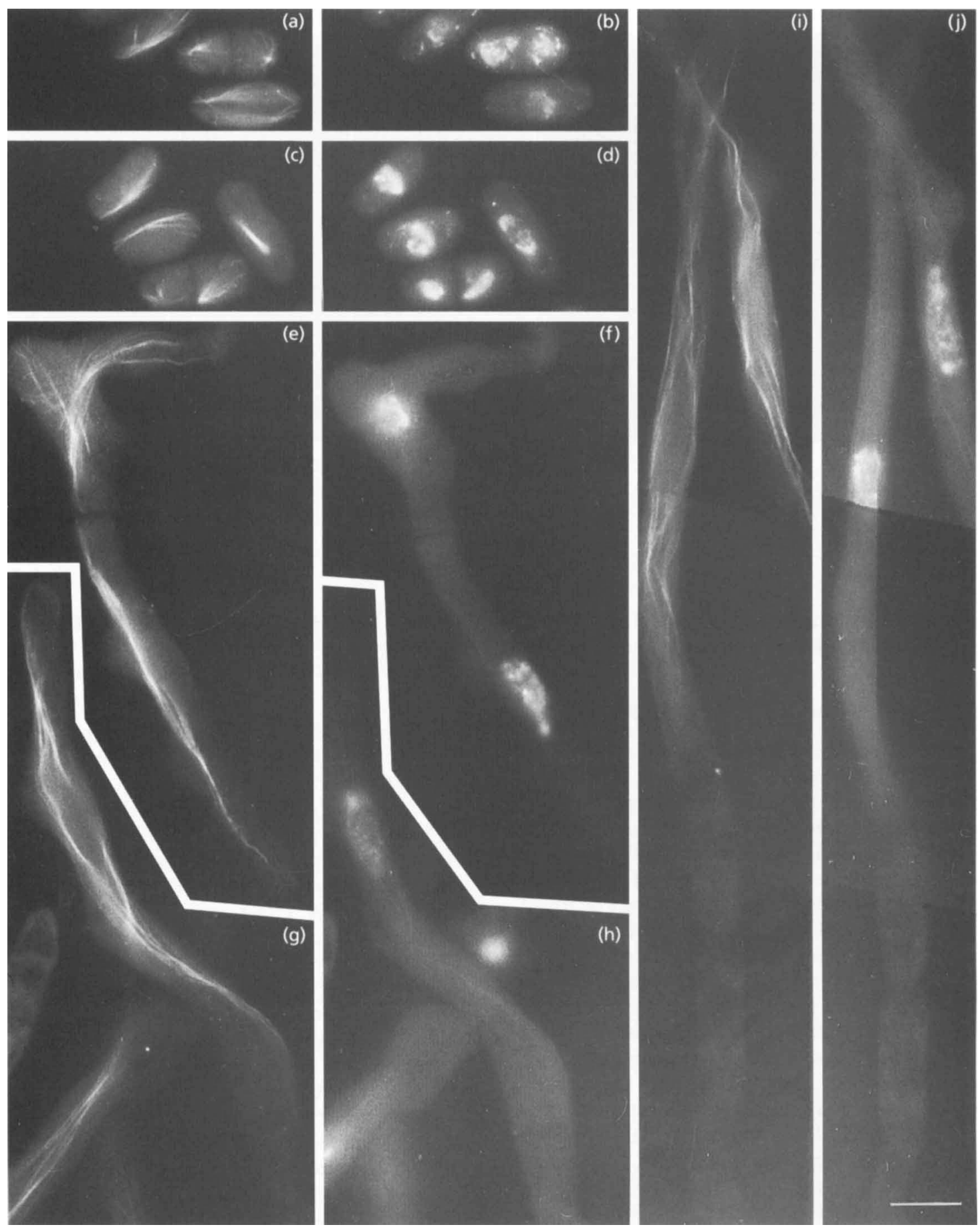

Fig. 5. Microtubular cytoskeleton in bipolarly growing yeasts $(a, c)$ and in unipolarly growing hyphae $(e, g, i) .(b, d, f, h$, j) DAPI-stained images of (a), (c), (e), (g) and (i), respectively. Bar, $6 \mu \mathrm{m}$.

gesting that not only the transition of yeasts to the mycelial phase but also the growth of the hyphae was sensitive to caffeine. To further examine the response of the mycelial phase to the presence of the drug, we grew the mycelium on YEMA plates ( $3 \mathrm{~mm}$ thick) for $10 \mathrm{~d}$ and then pipetted various amounts of caffeine solutions onto the medium $2-3 \mathrm{~mm}$ ahead of the growing hyphae.
Ten microlitres of $0 \cdot 1 \mathrm{M}$ solution caused a transient cessation (note the line in Fig. 8a), but $50 \mu$ of the $0.5 \mathrm{M}$ solution blocked hyphal extension definitively (Fig. 8b). Depending on the distance that separated the hyphae from the site of application of the latter solution, the hyphae died (Fig. 8d), converted to yeasts (Fig. 8e) or only underwent an intense septation and continued 

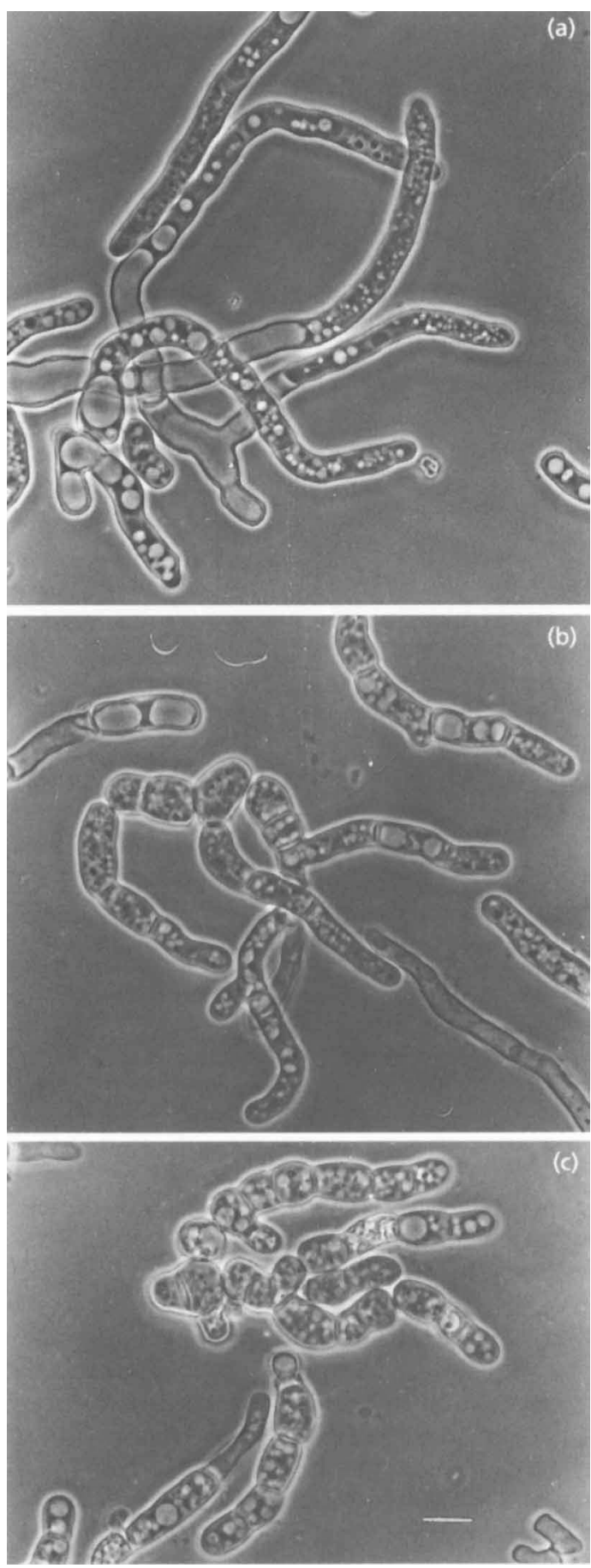

Fig. 6. Effect of osmotic pressure of the medium on the vacuolation and growth of cells. Yeasts grown on YMGA supplemented with (a) $0.1 \%$, (b) $0.4 \%$ and (c) $0.6 \%$ (w/v) $\mathrm{KCl}$, respectively. Bar, $5 \mu \mathrm{m}$.

growing as a dense, highly branched mycelium composed of short cells (Fig. 8f). Application of $5 \mu \mathrm{l} 0.03 \mathrm{M}$ cAMP solution also provoked septation and local thickening of mycelium, and $50 \mu \mathrm{l}$ of the $0 \cdot 1 \mathrm{M}$ solution halted the extension of hyphae. Return to yeast growth

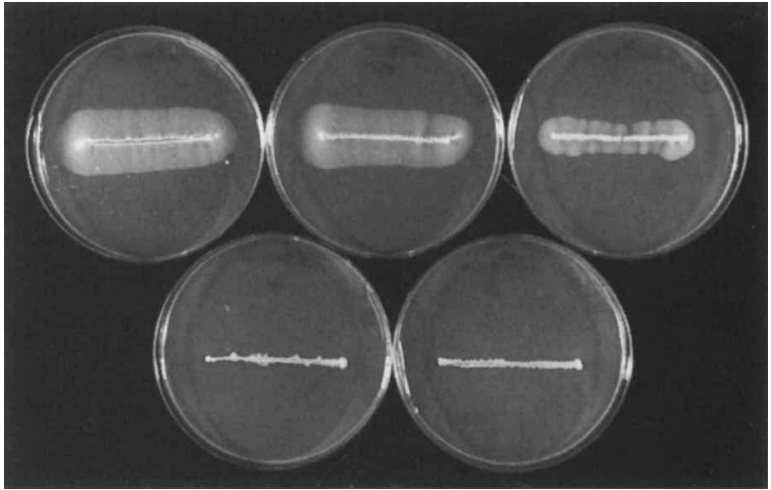

Fig. 7. Caffeine specifically inhibits mycelium formation. The concentration of caffeine in the plates was $0,0.2,0.4,0.5$ and $5.0 \mathrm{mM}$.

was also observed when smaller volumes of the latter solution were applied. Staining the nuclei of the fragmenting hyphae revealed that each fragment contained one nucleus (Fig. 9), which indicates that a nuclear division preceded each septum formation.

\section{Temperature-induced mycelium-to-yeast transition}

We reported in a recent paper (Sipiczki et al., 1998) that temperatures lower than $19^{\circ} \mathrm{C}$ or higher than $35^{\circ} \mathrm{C}$ prevented the yeast-to-mycelium transition, although yeast growth was not inhibited. Here we asked whether these temperatures allow hyphal growth once the mycelial phase has been established. To answer this question, we shifted 14-d-old mycelial cultures, grown on YEMA plates at $30^{\circ} \mathrm{C}$, to $18^{\circ} \mathrm{C}$ and $37^{\circ} \mathrm{C}$. Both temperatures halted the extension of the hyphae and provoked intense septation followed by fragmentation (Fig. 10a). The fragments separated and began to grow bipolarly. After several days incubation yeast microcolonies appeared at the front edge of the mycelium (Fig. $10 \mathrm{~b}, \mathrm{c})$, demonstrating that the effect of these temperatures was similar to that of caffeine and cAMP.

\section{DISCUSSION}

The results summarized in this study demonstrate that the phase transitions in the dimorphic cycle of Schiz. japonicus var. japonicus are associated with switches between unipolar and bipolar growth patterns. As demonstrated by the position of the calcofluor-positive cell wall regions and the distribution of actin dots, the yeast cells can grow at both cell ends. Under conditions which favour the formation of mycelial haloes, the yeast cells gradually switch to unipolar growth associated with an intense enlargement of vacuoles at the nongrowing pole and the block of cell separation after division. During the transition to mycelial growth, cell division becomes asymmetric: the mother cell divides to form a shorter cell with no large vacuole, which will be 

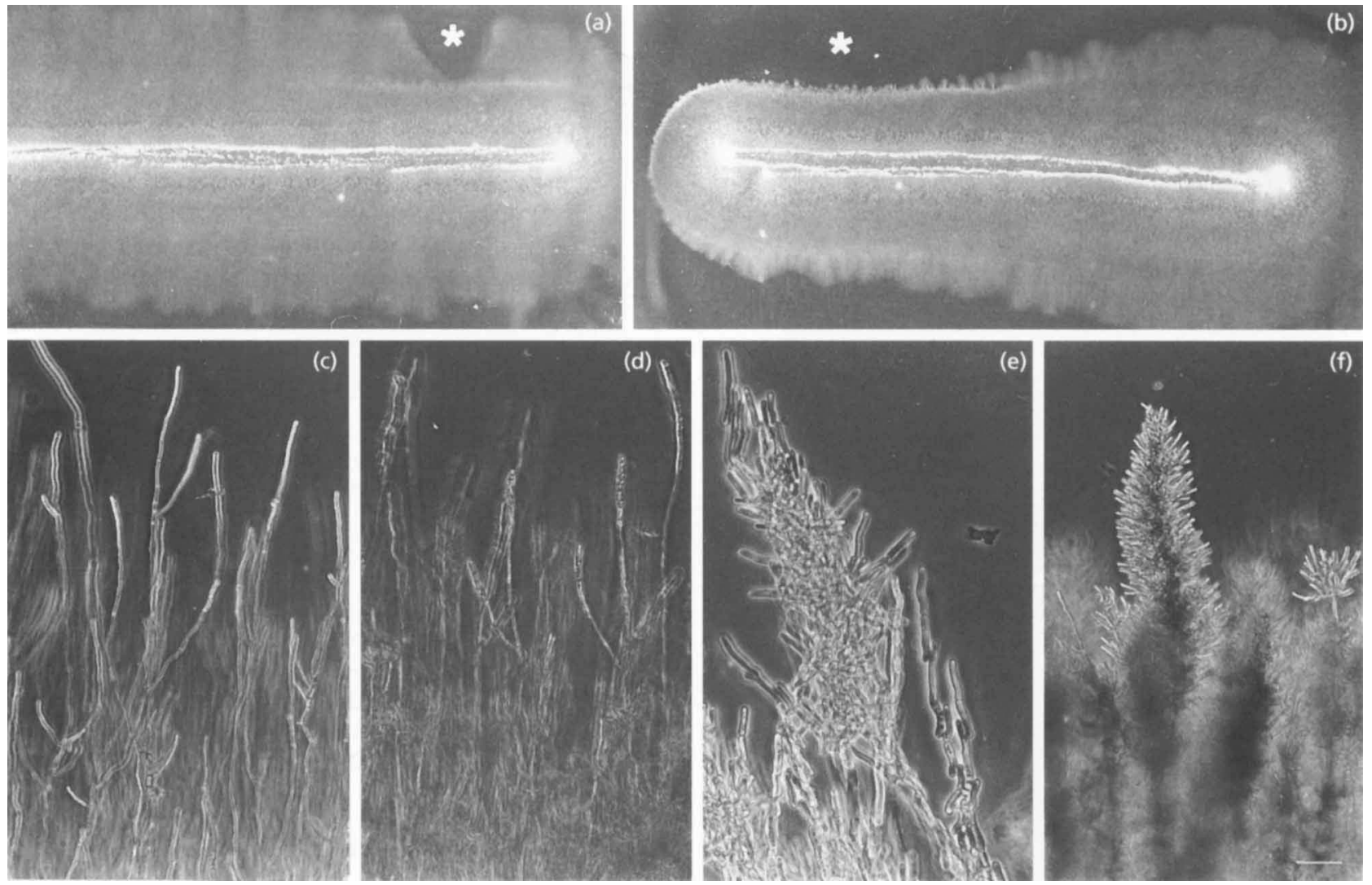

Fig. 8. Effect of caffeine on the mycelial phase. (a) Transient fragmentation (thick line) induced by caffeine added into the hole cut in the medium. (b) Inhibition of growth caused by $50 \mu 10.5 \mathrm{M}$ caffeine solution added onto the surface of the medium at the site marked. (c) Normal hyphae, (d) dead hyphae, (e) transition to yeast phase and (f) the formation of highly branched hyphae composed of short cells (see text for more detail). Bar, $35 \mu \mathrm{m}$.

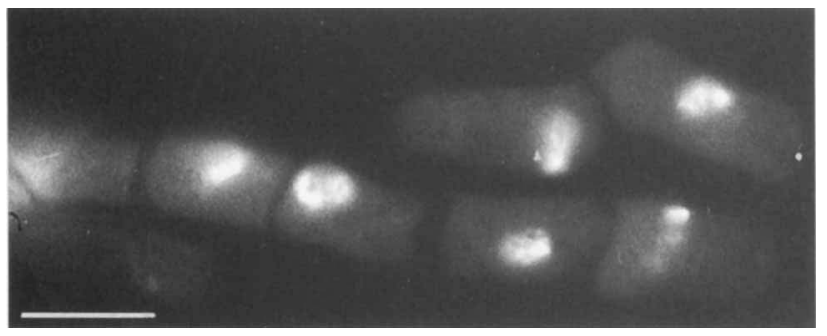

Fig. 9. Nuclei in the hyphae undergoing fragmentation upon exposure to $0.03 \mathrm{M}$ CAMP. Bar, $8 \mu \mathrm{m}$.

the tip cell of the hypha (Fig. 11). This cell will grow at its 'old' end, the end that also grew in the mother. The other daughter, which inherits the large vacuole, will grow at its 'new' end. Both daughters will divide asymmetrically again, producing one 'new-end-growing' (NEG) cell and one 'old-end-growing' (OEG) cell. In the yeast phase, cell divisions are more symmetric: the bipolarly growing cells produce daughters that show 'both-end-growing' (BEG) patterns. In the related fission yeast Schiz.pombe, in which dimorphism has not been reported, cells produce only BEG daughters, although the growth of one pole usually predominates (Mitchison \& Nurse, 1985; Miyata et al., 1986; Sipiczki et al., 1993). In the dimorphic strains of Sacch.cerevisiae a similar switch from bipolar to unipolar growth (budding) takes place during yeast-to-pseudomycelium transitions, but, by contrast to Schiz. japonicus var. japonicus, the yeast cells divide asymmetrically (large mother and small daughter) and the pseudohyphal cells divide symmetrically (equal size of mother and daughter) (Kron et al., 1994).

Although the switch to unipolar growth and the formation of large vacuoles occur simultaneously, the latter is not necessary for the former. Vacuolation can be inhibited without a concomitant inhibition of unipolar growth. The concentrations of glucose or caffeine that inhibit the enlargement of vacuoles still allow the formation of cell chains composed of unipolarly growing cells. What is the function of the vacuoles in the transition then? One possible role is to make the cell irreversibly committed to unipolar growth by 'silencing' one of its poles. The inability of the vacuolated cells to launch growth at the vacuolated ends upon shift to a fresh medium indicates that the large polar vacuole has 

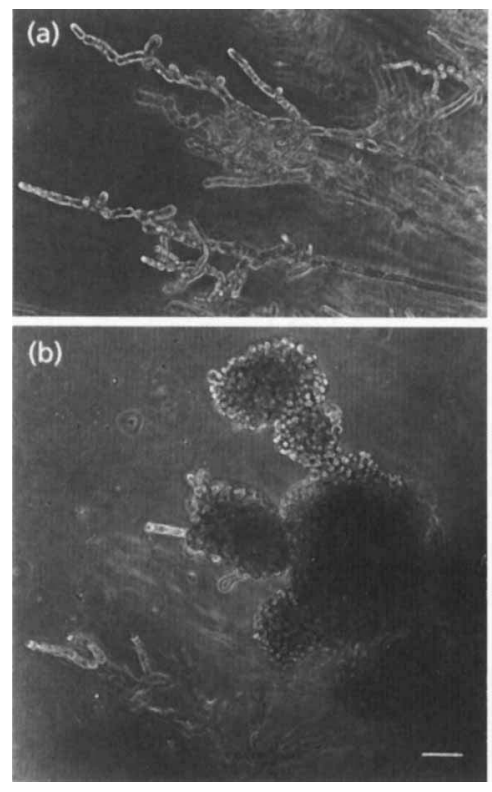

\section{(c)}

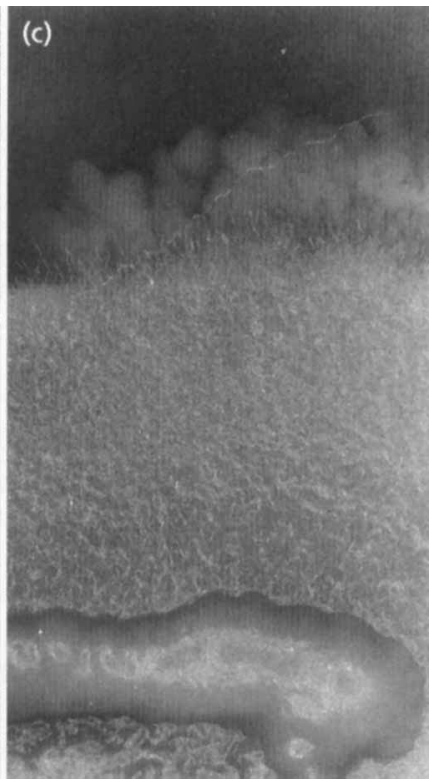

Fig. 10. Hypha-to-yeast transition upon shift to $37^{\circ} \mathrm{C}$. (a) Septation and fragmentation of hyphae. (b, c) Yeast microcolonies fringing a mycelium. Bar, $35 \mu \mathrm{m}$.

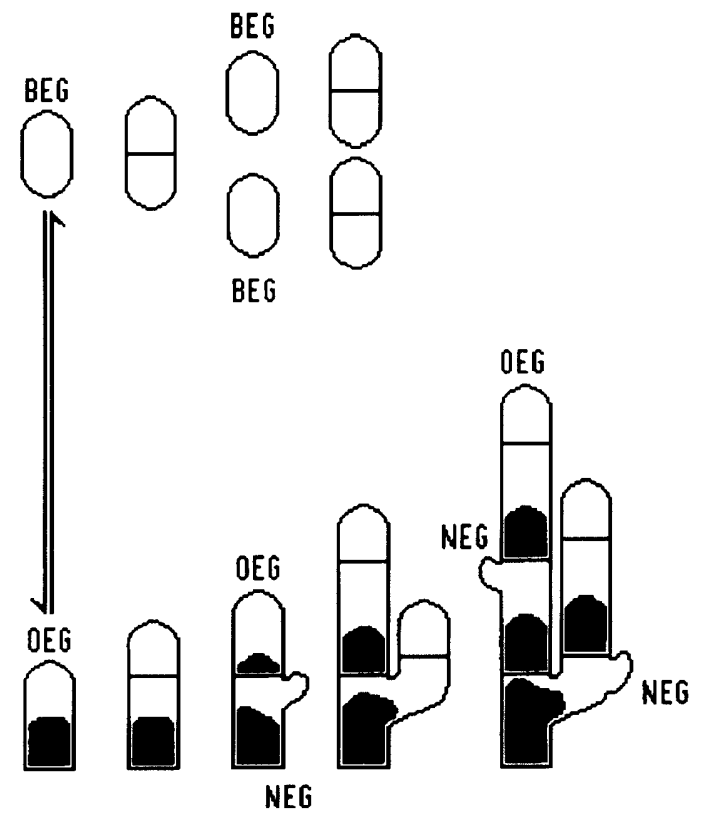

Fig. 11. Division symmetry in yeast phase and in mycelial phase. BEG, 'both-end-growing'; NEG, 'new-end-growing'; OEG, 'oldend-growing'. Dark areas represent vacuoles.

a 'growth silencing' effect. This is probably achieved by separating the cell tip from the bulk of the cytoplasm. The microtubules terminate at the vacuole and do not grow into the very thin cytoplasm that lies between the plasma membrane and the vacuole membrane (Sipiczki et al., 1998). The microtubular cytoskeleton is supposed to be involved in the intracellular organelle traffic (e.g. Terasaki \& Reese, 1994; Allan, 1995; Svoboda \& Slaninová, 1997). Since the polar vacuole prevents the microtubules from running to the pole, the organelles probably cannot be transported there. In the bipolarly growing yeast cells the bundles of microtubules run from pole to pole and thus can perform their functions at both cell ends. Another function of the polarly located vacuoles is the acceleration of cell extension: their rapid growth helps the cell extend rapidly. This acceleration is characteristic during the yeast-to-mycelium transition and then in the mycelial phase. In the latter case, the growth of the polar vacuoles is essential for the establishment of the invasive mycelium (Sipiczki et al., 1998). If cell chains are formed but their vacuolation is inhibited (e.g. on media with high osmotic pressure or caffeine), no invasive mycelium is formed. This role of vacuolation is reminiscent of that observed in the extension of germ tubes of C. albicans (Gow, 1994).

An important element of the yeast-to-mycelium transition is the failure of cell separation after septum formation. Whereas the bipolarly growing yeast cells always complete cytokinesis, the unipolarly extending cells are prone to leave their septa unsplit (true hyphae) or just partially cleaved (pseudohyphae). In Schiz. pombe, the cells of the mycelial mutant sep1-1 do not cleave their septa as long as they propagate but they do so in the last cell cycle before entering the stationary phase (Sipiczki et al., 1993). The inability of the propagating sep1-1 cells to complete cytokinesis was thought to be due to a temporal shift (delay) of cell division relative to karyokinesis. A similar shift might account for the failure of cell separation in Schiz. japonicus var. versatilis, as a result of the cell cycle reprogramming at the switch to unipolar growth.

In a recent paper (Sipiczki et al., 1998) we reported that solid substrates are indispensable for transition to the mycelial phase, and then the gradient of the nutrients (nitrogen) directs the hyphal extension in the medium. Here we show that the elevation of glucose concen- 
tration in the medium or a shift of the mycelium to temperatures of $37^{\circ} \mathrm{C}$ or $18{ }^{\circ} \mathrm{C}$ inhibits hyphal growth and can induce a return to the yeast phase.

In Schiz. pombe the addition of glucose to glucosestarved cells generates a transient increase of intracellular cAMP level which then falls to a level still significantly higher than the basal level (Byrne \& Hoffman, 1993). The increase results from the activation of adenylate cyclase. By analogy with Schiz. pombe, it is tempting to speculate that the high concentration of glucose in the medium might cause a constantly elevated cAMP level in the Schiz. japonicus var. japonicus cells, which then keeps the organism in the yeast phase. It is worth mentioning here that nitrogen starvation reduces the intracellular level of cAMP in Schiz. pombe (Maeda et al., 1990). Since nitrogen starvation was previously found to be necessary for the yeast-to-mycelium transition (Sipiczki et al., 1998), it can be supposed that the phase transitions in Schiz. japonicus var. japonicus are controlled by the increase and decrease of the cAMP level.

Consistent with this, caffeine also inhibits the yeast-tomycelium transition and provokes mycelium-to-yeast transition at concentrations not inhibitory to yeast growth. Since caffeine is an inhibitor of cAMP phosphodiesterase (Beach et al., 1985), these effects might also be attributed to an increase of intracellular cAMP level. cAMP added exogenously into the medium acts similarly. Thus, the transition to and the maintenance of the mycelial phase probably require a drastic fall in the intracellular cAMP level. Eukaryotic cells commonly respond to environmental signals by altering intracellular cAMP levels; for example, the dimorphic transitions in Mucor are also correlated with changes in cAMP (e.g. Larsen \& Sypherd, 1974; Orlowski \& Ross, 1981). Its yeast cells contained about four times the level of intracellular cAMP of the hyphal cells. During yeastto-hypha transition, there was a large decrease in cAMP levels, whereas the hypha-to-yeast transition was associated with a burst in cAMP synthesis. In the hemibasidiomycete Ustilago maydis the disruption of the uac1 gene (adenylate cyclase) resulted in a constitutively filamentous phenotype. Budding was restored upon growth in the presence of cAMP (Gold et al., 1994). Histoplasma capsulatum provides an example of the opposite relationship between cAMP level and morphology. In this organism Maresca et al. (1977) found about five times higher levels of cAMP in the mycelial phase than in the yeast phase. Caffeine can also alter the standard bipolar growth pattern of Schiz. pombe and make the cells grow more unipolarly, but cannot provoke a hypha-to-yeast transition in its filamentous mutant sep1-1 (Sipiczki \& Takeo, 1998).

We observed that the polar vacuolation is particularly sensitive to the increase of cAMP level. In the presence of caffeine or cAMP concentrations which inhibit the yeastto-hypha transition the yeast cells can switch to unipolar growth and form hyphal cell chains but do not undergo vacuolation. The mycelium exposed to these agents forms stubby hyphae composed of short, poorly vacu- olated cells. Thus, one possible target of the cAMPcontrolled regulation might be the process of vacuole enlargement.

On the basis of the findings presented in this and the previous paper (Sipiczki et al., 1998) we propose the following model. Schiz. japonicus var. japonicus forms bipolarly growing, symmetrically dividing (BEG) fission yeasts in liquid substrates and also on solid substrates as long as the intracellular cAMP level is high. With the local exhaustion of the nutrients (nitrogen source) in the solid substrate and the fall in the cAMP level, the yeast cells gradually switch to unipolar extension, develop large vacuoles, begin to divide asymmetrically (NEG and OEG cells) and abandon cell separation (septum cleavage). The resulting cell chains then develop into a mycelium. Due to the rapid extension of polar vacuoles, the hyphae of the mycelium can extend much faster than the yeast cells. The elimination of the nutrient (nitrogen source) gradient, the shift to temperatures restrictive for mycelial growth or the increase of the intracellular cAMP level induces a gradual transition to the yeast phase by inhibiting vacuole growth, activating cell separation and switching to bipolar (BEG) growth.

\section{ACKNOWLEDGEMENTS}

We thank Dr A. Kocková-Kratochvilová for the strain used in this study. We also thank I. Lakatos and Misako Ohkusu for technical assistance. This research was supported by grants provided by OTKA (Hungarian National Research Fund), the Hungarian Academy of Sciences and the Ministry of Education and Culture in Hungary.

\section{REFERENCES}

Alfa, C., Fantes, P., Hyams, J., McLeod, M. \& Warbrick, E. (1993). Experiments with Fission Yeasts. Cold Spring Harbor, NY: Cold Spring Harbor Laboratory.

Allan, V. (1995). Membrane traffic motors. FEBS Lett 369, 101-106.

Beach, D., Rodgers, L. \& Gould, J. (1985). RAN1 ${ }^{+}$controls the transition from mitotic division to meiosis in fission yeast. Curr Genet 10, 297-311.

Benko, Z. \& Sipiczki, M. (1992). Caffeine tolerance in Schizosaccharomyces pombe: physiological adaptation and interaction with theophylline. Can J Microbiol 39, 551-554.

Byrne, S. M. \& Hoffman, C. S. (1993). Six git genes encode a glucose-induced adenylate cyclase activation pathway in the fission yeast Schizosaccharomyces pombe. J Cell Sci 105, 10951100 .

Gimeno, C. J., Ljungdahl, P. O., Style, C. A. \& Fink, G. R. (1992). Unipolar cell divisions in the yeast $S$. cerevisiae lead to filamentous growth: regulation by starvation and RAS. Cell 68, 1077-1090.

Gold, S., Duncan, G., Barrett, K. \& Kronstad, J. (1994). cAMP regulates morphogenesis in the fungal pathogen Ustilago maydis. Genes Dev 8, 2805-2816.

Gow, N. A. R. (1994). Growth and guidance of the fungal hypha. Microbiology 140, 3193-3205.

Grallert, A., Miklos, I. \& Sipiczki, M. (1997). Division site selection, cell separation and formation of anucleate minicells in Schizosaccharomyces pombe mutants resistant to cell wall lytic enzymes. Protoplasma 198, 218-229. 
Hagan, I. M. \& Hyams, J. S. (1988). The use of cell division cycle mutants to investigate the control of microtubule distribution in the fission yeast Schizosaccharomyces pombe. J Cell Sci 89, 343-357.

Johnson, B. F., Calleja, G. B., Boisclair, I. \& Yoo, B. Y. (1979). Cell division in yeasts. III. The biased, asymmetric location of the septum in the fission yeast cell, Schizosaccharomyces pombe. Exp Cell Res 123, 253-259.

Kron, S. J., Styles, C. A. \& Fink, G. R. (1994). Symmetric cell division in pseudohyphae of the yeast Saccharomyces cerevisiae. Mol Biol Cell 5, 1003-1022.

Larsen, A. \& Sypherd, P. (1974). Cyclic adenosine $3^{\prime}, 5^{\prime}$-monophosphate and morphogenesis in Mucor racemosus. J Bacteriol $117,432-438$.

McDonald, I. J. \& Tsai, C. S. (1989). Continuous culture and intermediary carbon metabolism. In Molecular Biology of the Fission Yeast, pp. 367-396. Edited by A. Nasim, P. Young \& B. F. Johnson. San Diego: Academic Press.

Madhani, H. D. \& Fink, G. R. (1998). The riddle of MAP kinase signalling specificity. Trends Genet 14, 151-155.

Maeda, T., Mochizuki, N. \& Yamamoto, M. (1990). Adenylyl cyclase is dispensable for vegetative cell growth in the fission yeast Schizosaccharomyces pombe. Proc Natl Acad Sci USA 87, 7814-7818.

Maresca, B., Medoff, G., Schlessinger, D. \& Kobayashi, G. S. (1977). Regulation of dimorphism in the pathogenic fungus Histoplasma capsulatum. Nature 266, 447-448.

Marks, J. \& Hyams, J. S. (1985). Localization of F-actin through the cell division cycle of Schizosaccharomyces pombe. Eur J Cell Biol 39, 27-32.

Mitchison, J. M. \& Nurse, P. (1985). Growth in cell length in the fission yeast Schizosaccharomyces pombe. J Cell Sci 75, 357-376.

Miyata, H., Miyata, M. \& Johnson, B. F. (1986). Patterns of extension growth of the fission yeast, Schizosaccharomyces pombe. Can J Microbiol 32, 528-530.

Orlowski, M. \& Ross, J. F. (1981). Relationship of internal cyclic AMP levels, rates of protein synthesis and Mucor dimorphism. Arch Microbiol 129, 353-356.
Pancaldi, S., Dall'Olio, G., Poli, F., Fasulo, M. P. \& Vannini, G. L. (1989). Morphological changes induced by aniline blue in Schizosaccharomyces pombe. Microbios 57, 27-31.

Roberts, R. L. \& Fink, G. R. (1995). Elements of a single MAP kinase cascade in Saccharomyces cerevisiae mediate two developmental programs in the same cell type: mating and invasive growth. Genes Dev 8, 2974-2985.

Sabie, F. T. \& Gadd, G. M. (1992). Effect of nucleosides and nucleotides and the relationship between cellular adenosine $3^{\prime}: 5^{\prime}$ cyclic monophosphate (cyclic AMP) and germ tube formation in Candida albicans. Mycopathologia 119, 147-156.

Sipiczki, M. \& Ferenczy, L. (1977). Protoplast fusion of Schizosaccharomyces pombe auxotrophic mutants of identical matingtype. Mol Gen Genet 151, 77-81.

Sipiczki, M. \& Takeo, K. (1998). The effect of caffeine on cell cycle progression and polar growth in Schizosaccharomyces pombe. Biologia (Bratisl) 53, 295-300.

Sipiczki, M., Grallert, B. \& Miklos, I. (1993). Mycelial and syncytial growth in Schizosaccharomyces pombe induced by novel septation mutations. $J$ Cell Sci 104, 485-493.

Sipiczki, M., Takeo, K., Yamaguchi, M., Yoshida, S. \& Miklos, I. (1998). Environmentally controlled dimorphic cycle in a fission yeast. Microbiology 144, 1319-1330.

Svoboda, A. \& Slaninová, I. (1997). Colocalization of microtubules and mitochondria in the yeast Schizosaccharomyces japonicus var. versatilis. Can J Microbiol 43, 945-953.

Terasaki, M. \& Reese, T. S. (1994). Interactions among endoplasmic reticulum, microtubules, and retrograde movements of the cell surface. Cell Motil Cytoskelet 29, 291-300.

Yarrow, D. (1984). Genus 22. Saccharomyces Meyen ex Rees. In The Yeasts. A Taxonomic Study, pp. 379-395. Edited by N. J. W. Kreger-van Rij. Amsterdam: Elsevier.

Zaitsevskaya-Carter, T. \& Cooper, J. A. (1997). Spm1, a stressactivated MAP kinase that regulates morphogenesis in $S$. pombe. EMBO J 16, 1318-1331.

Received 3 June 1998; revised 17 August 1998; accepted 24 August 1998. 